\title{
Beiträge zur geographischen Verbreitung der Tenthrediniden.
}

Von Prof. P. Gabriel Strobl in Admont.

XIV. T he il. *)

\section{7ᄀ. Tenthredo L.}

rufiventris Fbr. (Ad. 456, Ht. 313). A i.: Melk, Donauau auf Gesträuch $(2 / 7 \quad 1$ \& mit schwarzer und 1 \& mit gelber Brust), im Stiftsgarten (19/6 $1 \sigma^{7}$ mit gelber Brust); Seitenstetten, auf Laub an Bachrändern $\left(22 / 5-7 / 7 \quad 1 \quad \sigma^{7}, 4\right.$ o und 1 \& mit gelbgeflecktem Schildchen.) - Erzgebirge (L a n g e 2 ㅇ). Sklo bei Lemberg (S. G. $\sigma^{7}$ ).

balteata Klg. (Ad. 447, Ht. 313). St.: Bei Admont, im Veitlgraben auf Petasites-Blättern $\left(5 / 7 \quad \sigma^{7}\right)$; Voralpenregion des Kalbling auf Alnus viridis (17/6 o); auf Heracleum am Natterriegel (1600 m, 24/6 \&).

(limbata Klg. Ad. 457 var.?) quadridens mihi. St.: Im Gesïuse (18/6 o). Stimmt genau nach Ad. bis auf die Fühler. Nach Ad. sind die Fühler schwarz, das 3. bis 5. Glied aber licht. Bei meinem o aber ist das 3. Glied nur an der Spitze unterseits weiss; das 4. ebenfalls nur unterseits weiss, aber bis gegen die Basis hin; das 5, weiss mit schwarzer Basalhälfte der Oberseite; das 6. ist ganz weiss; das 7. bis 8. oberseits schwärzlich, unterseits bräunlichweiss. Der Name quadridens bezieht sich auf den Kopfschild, da die schwarzen Ecken des ziemlich stark ausgerandeten, weissen Kopfschildes ebenfalls eine kleine (vielleicht individuelle) Ausrandung zeigen, so dass sich jederseits 2 kurze, stumpfe Zähne bilden. Wegen der auffallenden Färbung des 2. bis 5. Ringes nur mit limbata zu vergleichen und wohl eigene Art, (dann quadridens zu benennen). Das Geäder zeigt merkwürdige Abnormitäten, so besitzen z. B. die Vorderflügel 4 Radialzellen.

coryli Pz. (Ad. 447, Ht. 313 und intermedia Klg. Ht. 310, eine Var. des $\sigma^{\star}$ mit ganz schwarzen Fühlern). A i. : (Eb. $\left.\sigma^{\top}\right)$; Seitenstetten, an Bachrändern auf Sambucus und Dolden, Blümelsberghöhe auf Quercus $(13 / 5-18 / 6 \quad 3$ q). St.: Gesäuse, auf Berberis (30/5 q); Voralpen um Admont bis $1600 m$ auf Heracleum und Alnus viridis $\left(17 / 6-11 / 7 \quad 3\right.$ c und $1 \sigma^{7}$-var. intermedia). Prag (S. G. 2 \&).

*) XIII. Theil siehe pag. $275-281$.

Wiener Entomologische Zeitung, XV. Jahrg., X. Heft (10. December 1896). 
velox Fbr. (Ad. 449, Ht. 312, o'-biguttata Ht. 313 sec. Stein in Wien. Entom. Ztg. 1884, pag. 309). T i.: Monte Spinale bei Madonna di Campiglio (24,7 q). St.: Scheibleggerhochalpe bei Admont, auf Blumen (27/7 q, var.: die ganze Oberseite der Fühler ist dunkel, wie beim $\sigma^{7}$, sonst ganz normal, nach $\mathrm{S}$ t e i n).

v a r. alpicola Stein = var. simplex Zadd. sec. Kon. S t.: Scheibleggerhochalpe auf Blumen (27:7 \& determ. Kon.!); Krummholzwiesen des Kalbling (19/7 o); Vorclpenzaun der Kaiserau bei Admont ( $6 / 8$ o $)$; Hochschwung bei Rottenmann (1900 m 9/8, \&). Görz: Krnalpe (23/7 q). o sah ich nur im Hofmuseum aus Krain (1. M a $n \mathrm{n}$ ).

NB. Bei der Normalform $q$ ist der Hinterleib ganz schwarz; die Mitteltarsen sind gelb, mit breiter, schwarzer Räckenlinie; die Hintertarsen schwarz, nur das Endglied roth mit dunkler Rückenlinie, oder alle Glieder an der Basis roth, an der Spitze schwarz. Bei var. alpicola o aber ist der dritte bis incl. 6. Ring roth; die Mitteltarsen ohne deutliche dunkle Rückenlinie und die Hintertarsen roth mit breiter dunkJer Rückenlinie oder schwarzbraun mit rothen Gliederungen. Das Geäder ötters abnorm.

Lachnaniana Cam. (Ad. 453). A i.: (Eb. \&); Seitenstetten, auf Laub am Treflingbache ( $\sigma^{\top}$-poecilopus Mosc. Ad. 455).

rufipes Klg. (Ad. 458, Ht. 305). Var. Flügelschïppchen roth. A i.: (Eb. \&). St.: Admont, in einer Waldlichtung auf Blumen (24/7 \&).

atra L. (Ad. 440, Ht. 306). A i.: Melk, Donauleithen auf Abies excelsa (6/6 q); Seitenstetten, auf Abies, Tilia etc., $\sigma^{7}$ f nicht selten $(5 / 5-7 / 7$.) S t.: Admont, im Veitlgraben auf Alnus incana (Juni \&); Kaiserauer Voralpenwiesen (16/6 q); Jaring und Radkersburg auf Dolden (28/7, 7/8 2 q). - Prag (S. G. 2 \&). * Podolien (1. $\mathrm{H} \mathrm{o} \mathrm{ch} \mathrm{huth}$ \&).

v a r. dispar Klg. (Ad. 454, Ht. 306 als Art, Kon. Cat. als Var.; unterscheidet sich eigentlich nur durch das gelb gerandete Pronotum und den weissen Fleck über den Hinterhïften von atra, auch fehlt es nicbt an Uebergängen, daher die Zusammenziehung gerechtfertigt erscheint). A i.: (Eb. q); Melk, bei Winden einem Asılus germanicus abgejagt (2/6 o), bei Pöverding auf Dolden (23/6 o), an der Donau auf blühendem Crataegus (25/5 3 \&); Seitenstetten, aut Betula im Franzosen- 
wäldchen (10/5 오, $\sigma^{\star}$ mit schwarz gestreiften Schenkeln, wie bei atra $\left.\sigma^{7}\right)$; Stiftsgarten auf Tilia $\left(25 / 5 \sigma^{7}\right.$ q). S t.: Gesäuse, auf Berberis - Blüthen $\left(30 / 5 \sigma^{7}\right)$, Lichtmessberg bei Admont auf Abies und Alnus (Ende Juli $\sigma^{7}$ ). - Lemberg S. G. $९$ ).

var. Uebergangsform zu atıa: Der Fleck über den Hinterhüften klein, Schüppchen und Schenkelstreifen schwarz, Pronotum sehr schmal gelbgerandet oder ganz schwarz. A i.: Seitenstetten, im Franzosenwäldchen auf Abies (2/6 3 o $\left.\sigma^{\pi}\right)$, Blümelsberghöhe auf Quercus (19/5 $\left.\sigma^{7}\right)$.

colon Klg. (Ad. 445, Ht. 312). S t.: Im Veitlgraben bei Admont auf Salvia glutinosa (6:8 \& teste Kon.!); auf Krummholzwiesen des Kalbling $(5 / 7$ \&). Durch die glänzenden, nur zerstreut punktirten Brustseiten von velox und bipunctata sicher zu unterscheiden.

livida L. (Ad. 449, Ht. 312). A i.: Wien (Eb. ठ Melk, Donauau. Donauleithen, Winden, Wachberg auf Gesträuch, besonders Prunus avium $(15 / 6-11 / 7$ o $q)$; Seitenstetten, auf Laub $\sigma^{7} q$ ziemlich häufig $(25 / 5-7 / 7)$. St.: Auf grossblättrigen Waldpflanzen und auf Laub in Waldlichtungen um Admont, Johnsbach, Gstatterboden zerstreut (Juli, ơ). T i.: Innsbruck $\left(\sigma^{7}\right.$ ) ). - Kaplitz (S. G. $\left.\sigma^{7}\right)$. Lemberg $(4 / 6$ S. G. 2 \%; „frisst Psila fimetaria").

var. Hinterleibsende roth. A i.: Seitenstetten, auf Dolden im Franzosenwäldchen $(17 / 6$ o; die 3 letzten Ringe fast ganz roth). Olmütz (S. G. $₹$; die 4 letzten Ringe roth, auch der vorausgehende roth gefleckt).

solitaria Scp. (Rog. und Dalla Torre in Wien. Zool. Bot. Ges. 1882, pag. 596, fagi Pz. Ht. 312, maura Fbr. Ad. 462). A i.: Melk, am Wachberge auf Euphorbia cyp. $\left(7 / 50^{7}\right)$; Seitenstetten, auf Gebüsch an Bach- und Waldrändern (20/5-7/7 $2 \sigma^{7}, 5$ q). St.: Admont, auf Alnus incana (18/7 q). - Lemberg ( $7 / 6$ S. G. 2 q).

bipunctula Klg. (Ht. 312, Stein in Wien. Ent. Ztg. 1884, pag. 309, Kon. in Deutsch. Ent. Ztschrft. 1891, pag. 218 \&). S t.: Im Gesäuse auf Dolden (26/7 2 \&), auf Salvia glutinosa $\left(\begin{array}{lll}1 & 8 & \sigma^{7}\end{array}\right)$. G ̈̈ r z: St. Canzian auf Euphorbia nicaeensis $\left(24 / 7 \sigma^{7}\right)$. Meine $f$ unterscheiden sich von velox durch die Fühlerfarbe, das stärker ausgerandete. schwarze Epistom (nur je ein kleiner Seitenfleck ist weiss), die braune Oberlippe mit weissem Basalfleck, die zwar ebenfalls ganz matten, aber deutlich und dicht 
gekörnelten Brustseiten, den weissen Seitenfleck des 1. Hinterleibsringes, ganz schwarze Hinterbeine, schwarze Mittel- und grösstentheils schwarze Vorderschenkel. Die $\sigma^{x}$ unterscheiden sich von den o nur dadurch, dass die Seitenflecke des listom grösser sind, die Oberlippe ganz gelb ist und auch die Mittelschenkel - gleich den Vorderschenkeln - einen trübgelben Streifen besitzen.

mandibularis Pz. (Ad. 440, Ht. 305). A i.: (Eb. $\left.\sigma^{\top}\right)$; Melk, an der Bielach auf Petasites-Blättern (27 $\left.522 \sigma^{\top}\right)$. St.: Im Gesäuse Anfangs August auf Blumen, besonders Salvia glutinosa, $3 \sigma^{\top}, 1$ o; Admont, in einer Waldlichtung auf Salvia glutinosa (247

mesomelas L. (Ad. 460, viridis Klg. Ht. 310). Auf Laub (besonders Alnus incana), Abies excelsa, Dolden, grossblättrigen Wald- und Voralpenpflanzen, besonders Pteris aquilina und Adenostyles alpina, sehr häufig. A i.: Melk, Seitenstetten. S t. : Um Admont bis zur Krummholzgrenze überall, Gesäuse, Hohentauern. T i.: Innsbruck, Amras, Zirler Bergwiesen. C r t.: Oberdrauburg. - Lemberg, Karpathen (S. G. $1 \sigma^{7}, 6$ q, 1 q mit fast ganz schwarzen Hinterbeinen).

olivacea Klg. (Ad. 459, Ht. 309). S t.: Johnsbachgraben, auf Dolden (1/8 o); am Bösenstein um den Scheiplsee auf Alnus viridis (1700 m, 26/5, 1/8 2 q), sogar noch bei $2200 \mathrm{~m}$ (14/8 1 \&); am Hochschwung bei Rottenmann (1900 m, 19/8 q); am Rothkofel bei Turrach $(q)$.

flava Scp. (Ad. 445, flavicornis Vill. Ht. 311). Unter denselben Verhältnissen wie mesomelas und ebenso häufig, Mai bis August. A i.: Melk, Seitenstetten. St.: Admont bis auf die Voralpen, Gesäuse, Frohnleithen. T i. : Innsbruck, Amras, Selrain, Stubai, Zirler Bergwiesen. - Prag, Lemberg, Karpathen (S. G.).

\section{Schlussbemerknng.}

In diesen Beiträgen sind 454 Arten und 72 bemerkenswerthere $\mathrm{V}$ ari e tä t e $\mathrm{n}$ aufgeführt, von welchen auf $\mathrm{O}$ es terreich-Ungarn 400 Arten und $70 \mathrm{~V}$ ar. entfallen. Das am besten untersuchte Kronland $\mathrm{Nieder-Oesterreich}$ lieferte $316 \mathrm{Arten}$ und $49 \mathrm{~V}$ ar.; $\mathrm{St}$ ei ermark $162 \mathrm{Arten}$ und $24 \mathrm{Var}$. 


\section{$2 \mathrm{BHL}$ Biodiversity Heritage Library}

Strobl, Gabriel. 1896. "Beiträge zur geographischen Verbreitung der

Tenthrediniden. XIV. Theil." Wiener entomologische Zeitung 15, 295-298.

https://doi.org/10.5962/bhl.part.13886.

View This Item Online: $\underline{\text { https://www.biodiversitylibrary.org/item/43774 }}$

DOI: https://doi.org/10.5962/bhl.part.13886

Permalink: https://www.biodiversitylibrary.org/partpdf/13886

\section{Holding Institution}

Smithsonian Libraries

\section{Sponsored by}

Smithsonian

\section{Copyright \& Reuse}

Copyright Status: NOT_IN_COPYRIGHT

This document was created from content at the Biodiversity Heritage Library, the world's largest open access digital library for biodiversity literature and archives. Visit BHL at https://www.biodiversitylibrary.org. 